\title{
Report of the IAU/IAG Working Group on cartographic coordinates and rotational elements: 2006
}

\author{
P. Kenneth Seidelmann • B. A. Archinal • \\ M. F. A'hearn • A. Conrad • G. J. Consolmagno • \\ D. Hestroffer • J. L. Hilton • G. A. Krasinsky • \\ G. Neumann - J. Oberst - P. Stooke - E. F. Tedesco • \\ D. J. Tholen - P. C. Thomas • I. P. Williams
}

Received: 9 February 2007 / Accepted: 12 February 2007 / Published online: 3 July 2007

(C) Springer Science+Business Media B.V. 2007

\begin{abstract}
Every three years the IAU/IAG Working Group on Cartographic Coordinates and Rotational Elements revises tables giving the directions of the poles of rotation and the prime meridians of the planets, satellites, minor planets, and comets. This report introduces improved values for the pole and rotation rate of Pluto, Charon, and Phoebe, the pole of Jupiter, the sizes and shapes of Saturn satellites and Charon, and the poles, rotation rates, and sizes of some minor planets and comets. A high precision realization for the pole and rotation rate of the Moon is provided. The expression for the Sun's rotation has been changed to be consistent with the planets and to account for light travel time
\end{abstract}

Keywords Cartographic coordinates - Rotation axes · Rotation periods - Sizes · Shapes $\cdot$ Planets $\cdot$ Satellites $\cdot$ Minor planets $\cdot$ Comets

P. K. Seidelmann $(\bowtie)$

University of Virginia, Charlottesville, VA, USA

e-mail: pks6n@virginia.edu

B. A. Archinal

U.S. Geological Survey, Flagstaff, AZ, USA

M. F. A'hearn

University of Maryland, College Park, MD, USA

A. Conrad

W. M. Keck Observatory, Kamuela, HI, USA

G. J. Consolmagno

Vatican Observatory, Vatican City State, Italy

D. Hestroffer

IMCCE, Paris Observatory, CNRS, Paris, France

J. L. Hilton

U.S. Naval Observatory, Washington DC, USA 


\section{Introduction}

The IAU Working Group on Cartographic Coordinates and Rotational Elements of the Planets and Satellites was established as a consequence of resolutions adopted by Commissions 4 and 16 at the IAU General Assembly at Grenoble in 1976. The Working Group became a joint working group of the IAU and the International Association of Geodesy (IAG) in 1985. Now within the IAU the working group is a joint working group of Divisions I and III, and not part of commissions. The first report of the Working Group was presented to the General Assembly at Montreal in 1979 and published in the Trans. IAU 17B, 72-79, 1980. The report with appendices was published in Celestial Mechanics 22, 205-230, 1980. The guiding principles and conventions that were adopted by the Group and the rationale for their acceptance were presented in that report and its appendices. The second report of the Working Group was published in the Trans. IAU 18B, 151-162, 1983, and also in Celestial Mechanics 29, 309-321, 1983. In 2003 the name of the Working Group was shortened to the Working Group on Cartographic Coordinates and Rotational Elements. The table summarizes the references to all the reports.

\begin{tabular}{|c|c|c|}
\hline Report & General Assembly & Celestial Mechanics and Dynamical Astronomy \\
\hline 1 & Montreal in 1979 & 22, 205-230 (Davies et al. 1980). \\
\hline 2 & Patras in 1982 & 29, 309-321 (Davies et al. 1983). \\
\hline 3 & New Delhi in 1985 & 39, 103-113 (Davies et al. 1986). \\
\hline 4 & Baltimore in 1988 & 46, 187-204 (Davies et al. 1989). \\
\hline 5 & Buenos Aires in 1991 & 53, 377-397 (Davies et al. 1992). \\
\hline 6 & Hague in 1994 & 63, $127-148$ (Davies et al. 1996). \\
\hline 7 & Kyoto in 1997 & No report \\
\hline 8 & Manchester in 2000 & 82, 83-110 (Seidelmann et al. 2002). \\
\hline 9 & Sydney in 2003 & 91, 203-215 (Seidelmann et al. 2005). \\
\hline 10 & Prague in 2006 & This paper \\
\hline
\end{tabular}

G. A. Krasinsky

Institute for Applied Astronomy, St. Petersburg, Russia

G. Neumann

NASA Goddard Space Flight Center, Greenbelt, MD, USA

J. Oberst

DLR Berlin Adlershof, Berlin, Germany

P. Stooke

University of Western Ontario, London, Canada

E. F. Tedesco

University of New Hampshire, Durham, NH, USA

D. J. Tholen

University of Hawaii, Honolulu, HI, USA

P. C. Thomas

Cornell University, Ithaca, NY, USA

I. P. Williams

Queen Mary, University of London, London, UK

盽 Springer 
Reprints and preprints of the previous and this report can be found at the working group web site: http://astrogeology.usgs.gov/Projects/WGCCRE. Previous reports are also available at the web site: http://www.springerlink.com/content/100246.

The previous report introduced and recommended a consistent system of coordinates for both minor planets and comets. This system is not the same as the system for planets and satellites, which is not being changed. Pluto is included, as in the past, in the system of planets. It is recognized that the existence of two different systems has the potential for confusion, but the methods required for minor planets and comets differ sufficiently to justify the use of two different systems. This report includes descriptions of the two systems; one for planets and satellites and another for minor planets and comets. The use of a uniform system for minor planets and comets is highly recommended.

The IAU passed Resolution 5A at the General Assembly in Prague on August 24, 2006, adopting a definition of a planet, which changes the classification of Pluto and some other solar system bodies. This report, which is based on the progress of the past triennium, has retained the previous classifications of solar system bodies. Future versions of the report will incorporate changes as appropriate.

At the IAU General Assembly in Prague Brent Archinal was elected as the new and third chairman of this Working Group.

\section{Definition of rotational elements for planets and satellites}

Planetary coordinate systems are defined relative to their mean axis of rotation and various definitions of longitude depending on the body. The longitude systems of most of those bodies with observable rigid surfaces have been defined by references to a surface feature such as a crater. Approximate expressions for these rotational elements with respect to the International Celestial Reference Frame (ICRF) (Ma et al. 1998) have been derived. The ICRF is the reference frame of the International Celestial Reference System and is itself epochless. There is a small (well under 0.1 arcsecond) rotation between the ICRF and the mean dynamical frame of J2000.0. The epoch J2000.0, which is JD 2451545.0 (2000 January 11200 hours), Barycentric Dynamical Time (TDB), is the epoch for variable quantities, which are expressed in units of days ( 86400 SI seconds) or Julian centuries of 36525 days. TDB is the reference time scale for time dependent variables. TDB was clarified in definition at the IAU General Assembly of 2006 in Prague. TDB, sometimes called $\mathrm{T}_{\text {eph }}$, is roughly equivalent to Terrestrial Time (TT) in epoch and rate. UTC, TCB, and TCG differ from TT in epoch and rate. For more information on reference systems and time scales see Kovalevsky and Seidelmann (2004), http://www.iers.org, http://rorf.usno.navy.mil/ICRF/, or http://aa.usno.navy.mil/faq/docs/ICRS_doc.php.

The north pole is that pole of rotation that lies on the north side of the invariable plane of the solar system. The direction of the north pole is specified by the value of its right ascension $\alpha_{0}$ and declination $\delta_{0}$. With the pole so specified, the two intersection points of the body's equator and the ICRF equator are $\alpha_{0} \pm 90^{\circ}$. We chose one of these, $\alpha_{0}+90^{\circ}$, and define it as the node $Q$. Suppose the prime meridian has been chosen so that it crosses the body's equator at point B. We then specify the location of the prime meridian by providing a value for $W$, the angle measured easterly along the body's equator between the node $Q$ and the point B (see Fig. 1). The right ascension of the point $Q$ is $90^{\circ}+\alpha_{0}$ and the inclination of the planet's equator to the celestial equator 
Fig. 1 Reference system used to define orientation of the planets and their satellites

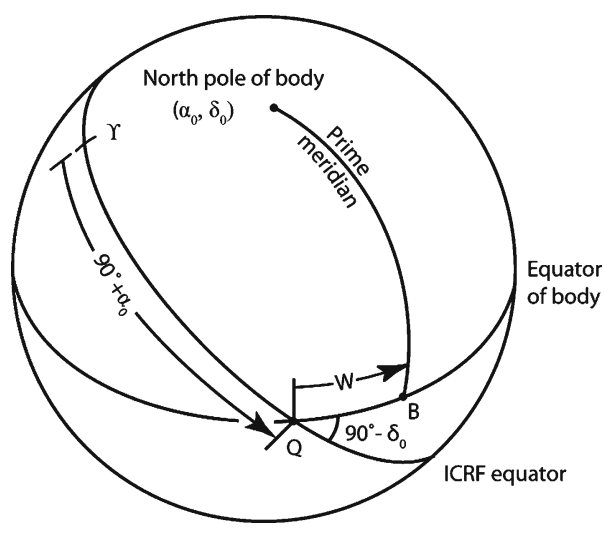

is $90^{\circ}-\delta_{0}$. As long as the planet, and hence its prime meridian, rotates uniformly, $W$ varies nearly linearly with time. In addition, $\alpha_{0}, \delta_{0}$, and $W$ may vary with time due to a precession of the axis of rotation of the planet (or satellite). If $W$ increases with time, the planet has a direct (or prograde) rotation, and, if $W$ decreases with time, the rotation is said to be retrograde.

In the absence of other information, the axis of rotation is assumed to be normal to the mean orbital plane of the planet or the satellite; Mercury ${ }^{1}$ and most of the satellites are in this category. For many of the satellites, it is assumed that the rotation rate is equal to the mean orbital period (i.e. synchronous rotation, but in some cases such an assumption still needs to be validated).

The angle $W$ specifies the ephemeris position of the prime meridian. For planets or satellites without any accurately observable fixed surface features, the adopted expression for $W$ defines the prime meridian and is not subject to correction for this reason. However, the rotation rate may be redefined for other reasons. Where possible, however, the cartographic position of the prime meridian is defined by a suitable observable feature, and so the constants in the expression $W=W_{0}+\dot{W} d$, where $d$ is the interval in days from the standard epoch, are chosen so that the ephemeris position follows the motion of the cartographic position as closely as possible; in these cases the expression for $W$ may require emendation in the future.

Recommended values of the constants in the expressions for $\alpha_{0}, \delta_{0}$, and $W$, in celestial equatorial coordinates, are given for the planets and satellites in Tables 1 and 2. In general, these expressions should be accurate to one-tenth of a degree; however, two decimal places are given to assure consistency when changing coordinates systems. Zeros have sometimes been added to rate values $(\dot{W})$ for computational consistency and are not an indication of significant accuracy. Additional decimal places are given in the expressions for Mercury, the Moon, Mars, Saturn, and Uranus, reflecting the greater confidence in their accuracy. Expressions for the Sun and Earth are given to a similar precision as those of the other bodies of the solar system and are for comparative purposes only.

1 Based on dynamical arguments, e.g. Peale (2006), Mercury's obliquity is thought to be no more than a few arcseconds; however, the current uncertainty in its obliquity is several degrees (J. L. Margot 2003 , private communication). 
Table 1 Recommended values for the direction of the north pole of rotation and the prime meridian of the Sun and planets

$\alpha_{0}, \delta_{0}$ are ICRF equatorial coordinates at epoch J2000.0.

Approximate coordinates of the north pole of the invariable plane are $\alpha_{0}=273^{\circ} .85, \delta_{0}=66^{\circ} .99$.

$T=$ interval in Julian centuries (of 36525 days) from the standard epoch

$d=$ interval in days from the standard epoch.

The standard epoch is JD 2451545.0, i.e. 2000 January 112 hours TDB

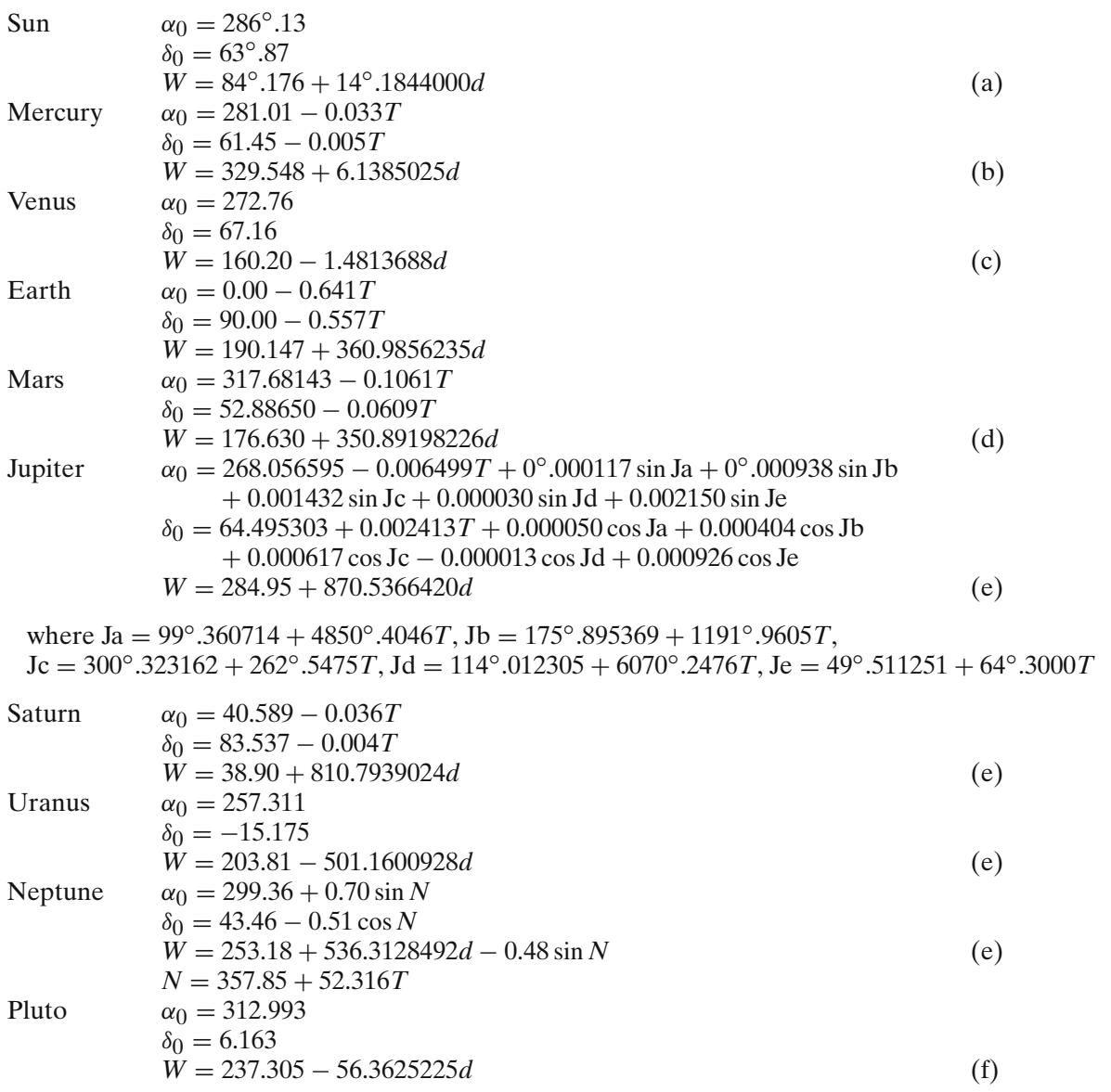

(a) The equation $W$ for the Sun is now corrected for light travel time and removing the aberration correction. See Appendix

(b) The $20^{\circ}$ meridian is defined by the crater Hun Kal

(c) The $0^{\circ}$ meridian is defined by the central peak in the crater Ariadne

(d) The $0^{\circ}$ meridian is defined by the crater Airy- 0

(e) The equations for $W$ for Jupiter, Saturn, Uranus and Neptune refer to the rotation of their magnetic fields (System III). On Jupiter, System I $\left(W_{I}=67^{\circ} .1+877^{\circ} .900 d\right)$ refers to the mean atmospheric equatorial rotation; System II $\left(W_{I I}=43^{\circ} .3+870^{\circ} .270 d\right)$ refers to the mean atmospheric rotation north of the south component of the north equatorial belt, and south of the north component of the south equatorial belt

(f) The $0^{\circ}$ meridian is defined as the mean sub-Charon meridian 


\section{Coordinate system for the moon}

The recommended coordinate system for the Moon is the mean Earth/polar axis (ME) system. This is in contrast to the principal axis (PA) system, sometimes called the axis of figure system. The ME system, sometimes called the mean Earth/rotation axes system, is recommended because nearly all cartographic products of the past and present have been aligned to it (Davies and Colvin 2000). The difference in the coordinates of a point on the surface of the Moon between these systems is approximately $860 \mathrm{~m}$. In past reports the rotation and pole position for the Moon have been given for the ME system using closed formulae. For convenience for many users, those formulae are repeated here in Table 2 . However, users should note that these are valid only to the approximately $150 \mathrm{~m}$ level of accuracy, as shown e.g. by Konopliv et al. (2001, Fig. 3). For high precision work involving e.g. spacecraft operations, high-resolution mapping, and gravity field determination, it is recommended that a lunar ephemeris be used to obtain the libration angles for the Moon from which the pole position and rotation can be derived.

Specifically, the NASA/JPL DE403/LE403 (Developmental Ephemeris 403/Lunar Ephemeris 403), commonly known as DE403, is considered the best currently available lunar ephemeris. See the website http://ssd.jpl.nasa.gov/iau-comm4/ for more information on the DE403 and how to obtain a copy. The development of a new JPL lunar ephemeris is under consideration (E. M. Standish et al. 2007, private communciation) and, if it does become available, it might be used for the highest possible accuracy. Polynomial representations of the (Euler) lunar libration angles and their rates in the PA system are stored in the ephemeris file. These three libration angles are:

(a) $\varphi$, the angle along the ICRF equator, from the ICRF X-axis to the ascending node of the lunar equator;

(b) $\theta$, the inclination of the lunar equator to the ICRF equator; and

(c) $\psi$, the angle along the lunar equator from the node to the lunar prime meridian.

Coordinates or Euler angles in the ME system (vector $\mathrm{M}$ ) can be rotated to the PA system (vector P) using the following expression (Konopliv et al. 2001, p. 7):

$$
\mathrm{P}=\mathrm{R}_{\mathrm{Z}}\left(63^{\prime \prime} .8986\right) \mathrm{R}_{\mathrm{y}}\left(79^{\prime \prime} .0768\right) \mathrm{R}_{\mathrm{x}}\left(0^{\prime \prime} .1462\right) \mathrm{M}
$$

Conversely, coordinates or Euler angles in the PA system can be rotated into the ME system with:

$$
\mathrm{M}=\mathrm{R}_{\mathrm{x}}\left(-0^{\prime \prime} .1462\right) \mathrm{R}_{\mathrm{y}}\left(-79^{\prime \prime} .0768\right) \mathrm{R}_{\mathrm{z}}\left(-63^{\prime \prime} .8986\right) \mathrm{P}
$$

where $R_{x}, R_{y}$, and $R_{z}$ are the standard rotation matrices for right-handed rotations around the $\mathrm{X}, \mathrm{Y}$, and $\mathrm{Z}$ axes respectively.

Therefore, for a given epoch, the user should obtain $\varphi, \theta$, and $\psi$ from the ephemeris file and store them as the vector P, apply the transformation in Eq. 2, and extract the angles, now in the ME system from the vector $M$. These angles can then be converted with:

$$
\begin{aligned}
& \alpha_{0}=\varphi-90^{\circ} \\
& \delta_{0}=90^{\circ}-\theta \\
& W=\psi
\end{aligned}
$$


త్తి

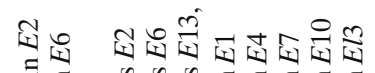

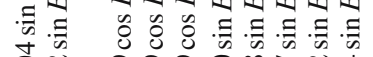

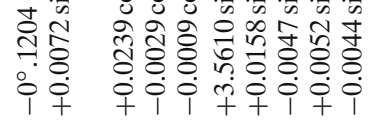

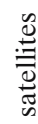

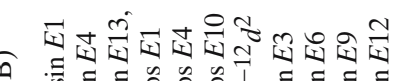

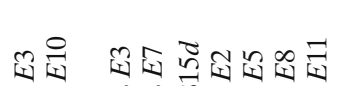
两过

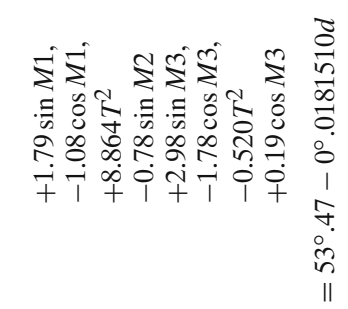

$+2 \hat{\square}$

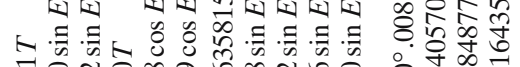

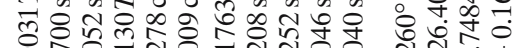

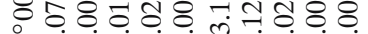

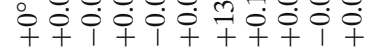

II $++\frac{+}{6}$ 凷

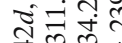
के ला 笔过这四

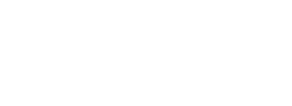

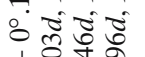

1 过

के

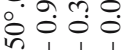
in +++ ॥ ลे ڤี

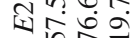
बृत ลี \|\|$\|$

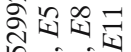
ใิ ○ ํํํ T)

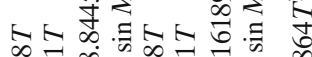

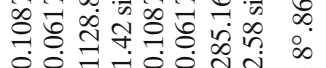
1)

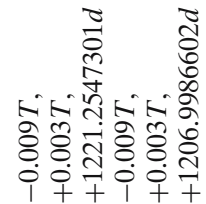

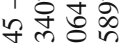

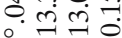
in ++1

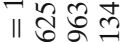
邽它它的 एँ $\|$ \|

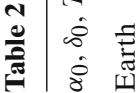
过国国 
$\hat{e}$

e

क्व

รี

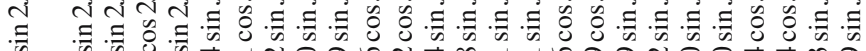
क

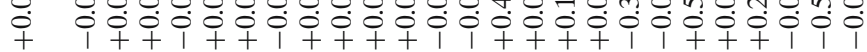

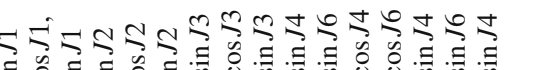

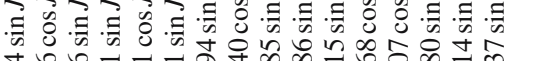

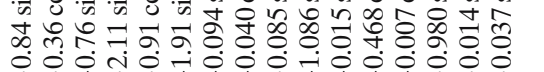

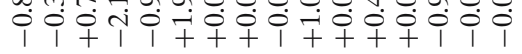

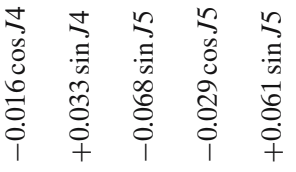

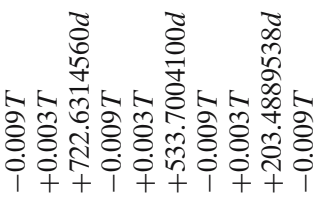

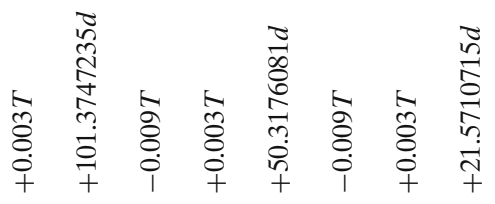
: 足 \&

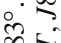
iิ

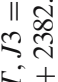

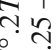

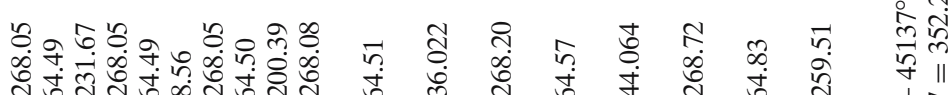

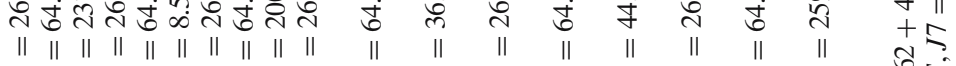
\| \| \| \| \| \|

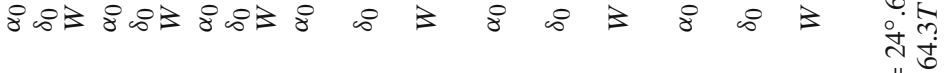

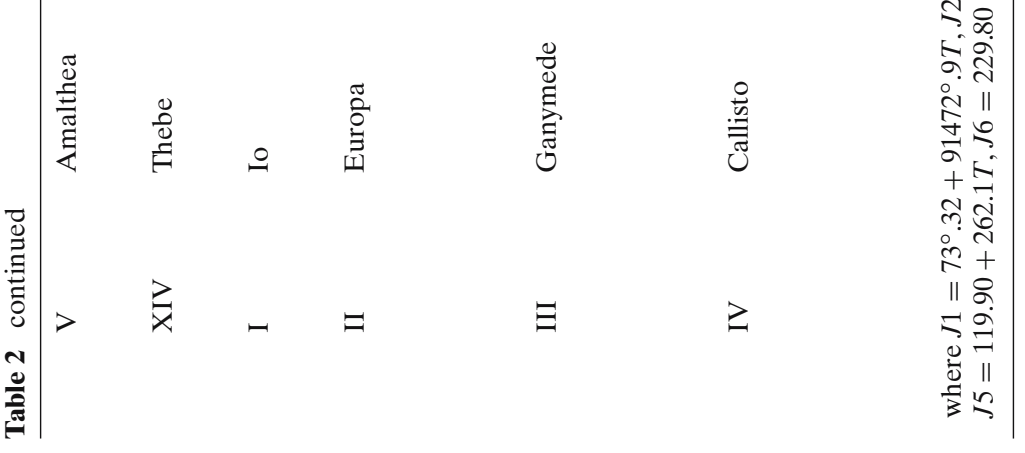




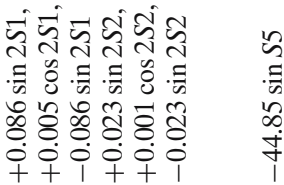

Ð

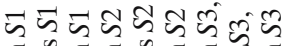

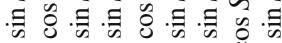
กं की

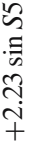

mitititi

迥芯芯

零 总

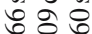

$+$

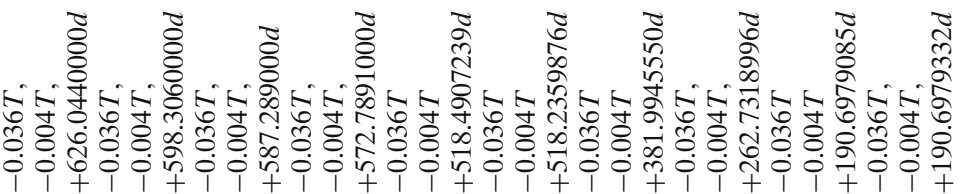

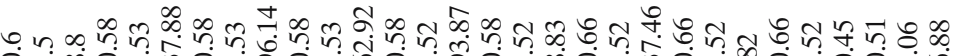

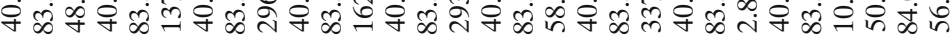
|| || || || || || || || || || || || || || || || || || || || || || || || || || || || || ||

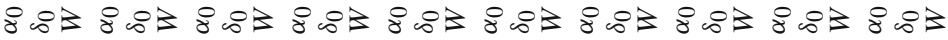

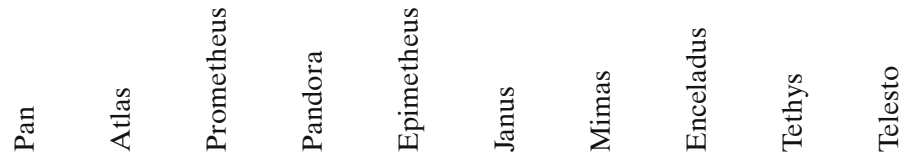

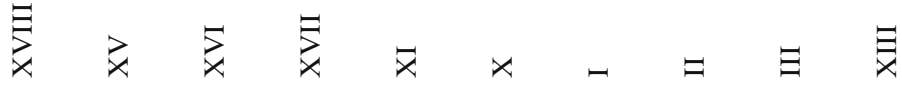

㠩 

e $)$
$\hat{\sigma}$
$\sqrt{2}$

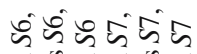

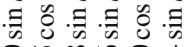

을 용요 웡

iि

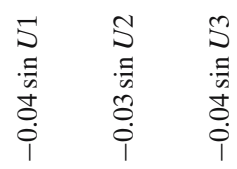

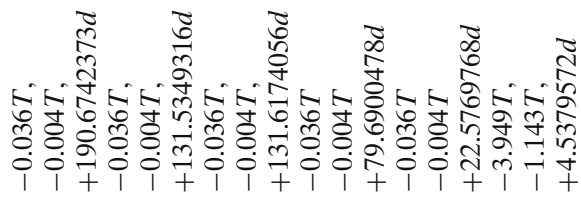

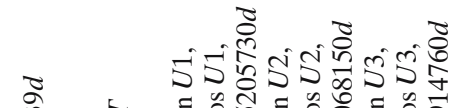

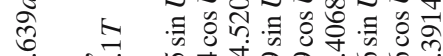

in

요

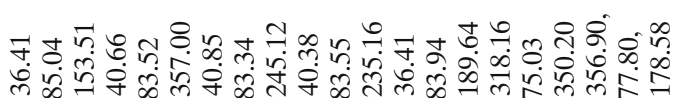

$\stackrel{10}{\circ}$

m.

|| || || || || || || || || || || || || || || || || || || || ||

$\| \stackrel{2}{\circ}$

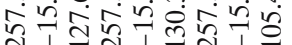

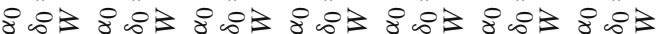

ڤ. | || || || || || || ||

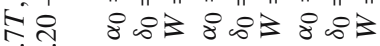

○ं के

in

$+\infty$

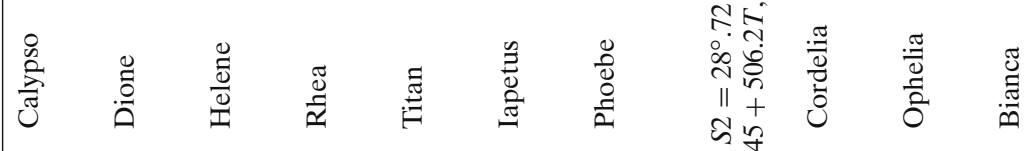

ㄱ.

iे

点的

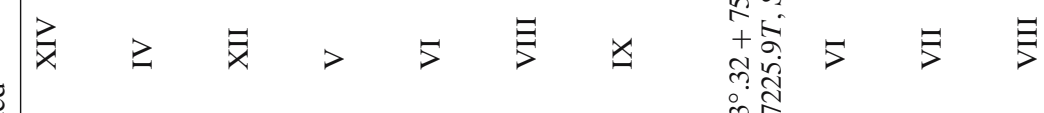

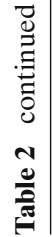

mก

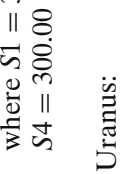

祭 Springer 


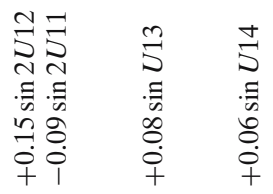

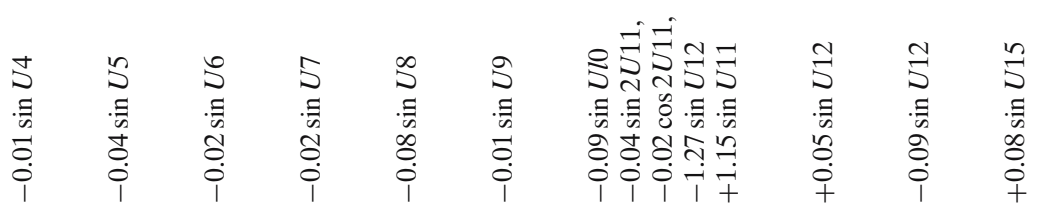

过选

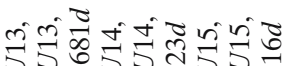

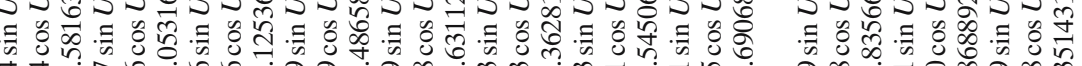

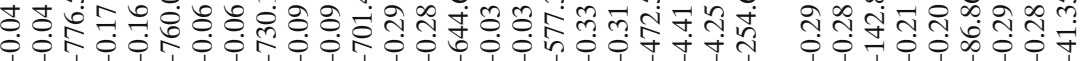

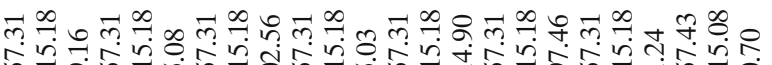

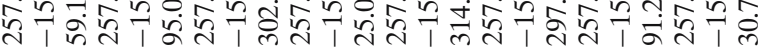
|| || || || || || || || || || || || || || || || || || || || || || || ||

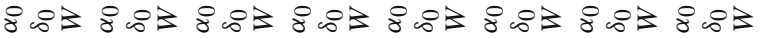

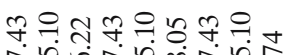

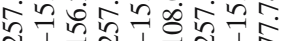
|| || || || || || || || || $8038 \sin 8$

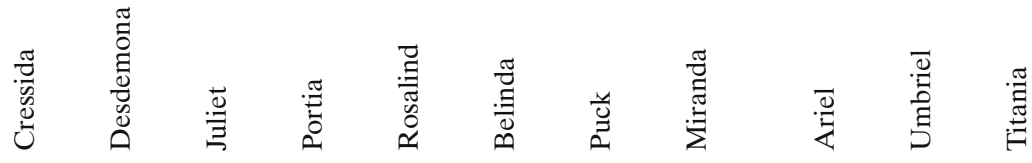

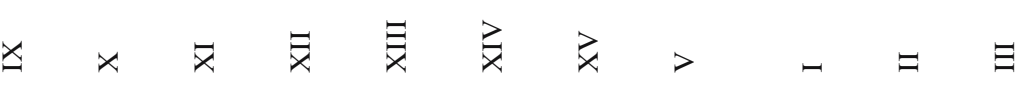




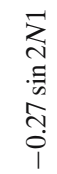

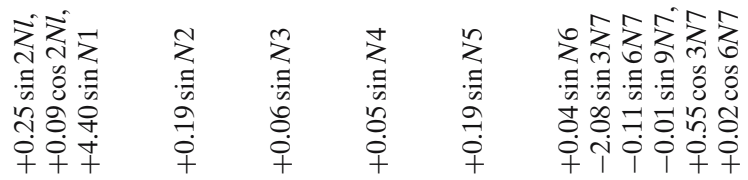

0
5
an
0
0
0
+

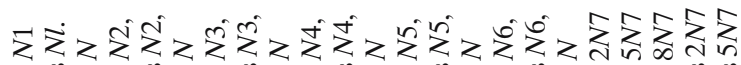

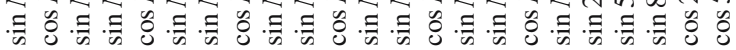

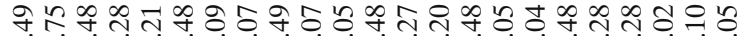

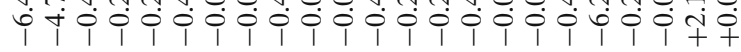

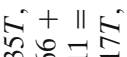

mำ 소

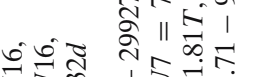
$\sin$ $+5 \dot{0} 0$ कर्क ले

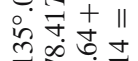
a 0 ก

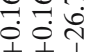
तN $\infty$ $3+\| \frac{1}{2}$ $\rightarrow \infty \equiv$ in

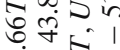

7.

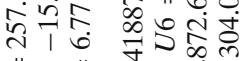
\| $\|$ ||

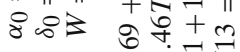
㠻守-

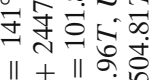

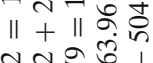

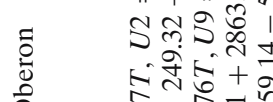

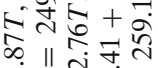
$\circ$ in ริ + กิำำ

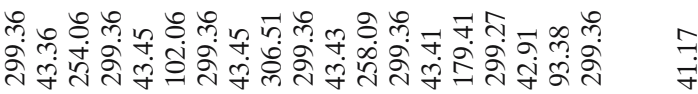
|| || || || || || || || || || || || || || || || || || || ||

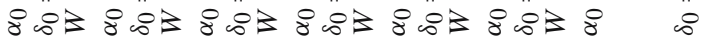

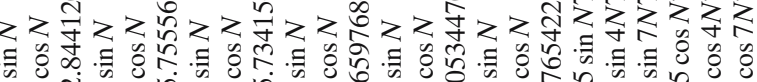

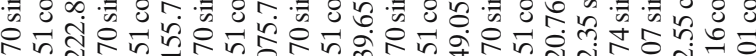

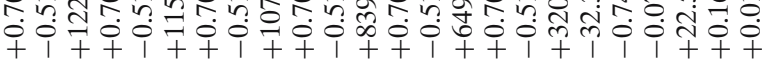

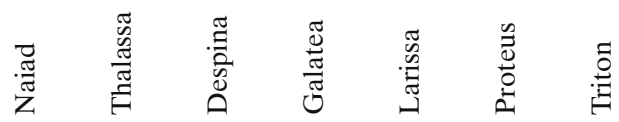
ヨ 


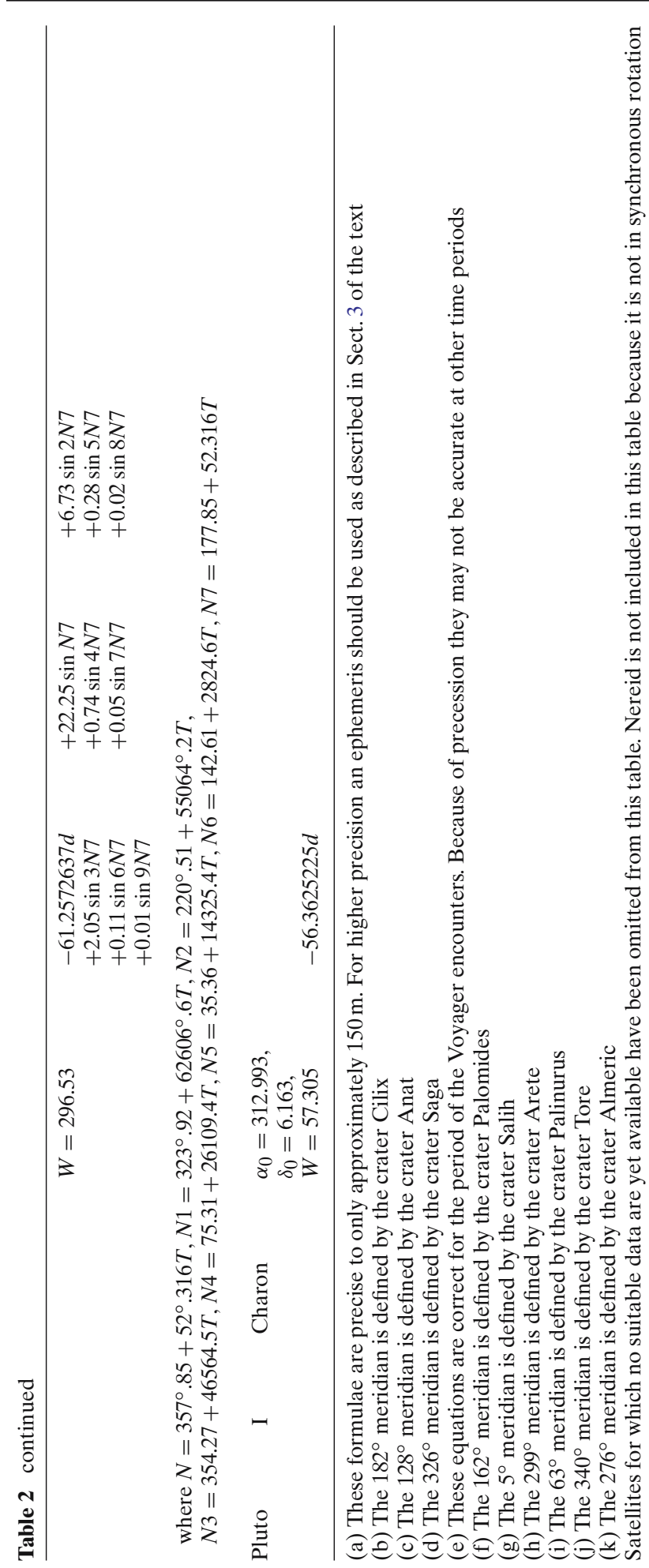


giving the lunar rotation angles in the standard $\alpha_{0}, \delta_{0}$, and $W$ formulation (of Table 2) and in the ME system.

Alternatively, if the user has coordinates for a point in ICRF coordinates (vector I) that they wish to convert to ME coordinates, for a given epoch the user should obtain $\varphi, \theta$, and $\psi$ from the ephemeris file, and then do the conversion:

$$
M=R_{x}\left(-0^{\prime \prime} .1462\right) R_{y}\left(-79^{\prime \prime} .0768\right) R_{Z}\left(-63^{\prime \prime} .8986\right) R_{Z}(\psi) R_{x}(\theta) R_{Z}(\varphi) I
$$

with $\mathrm{M}$ now being the coordinates of the point in the ME system. The user should note that the numerical values for the rotations in Eqs. 1, 2, and 3 are specific to DE403 and are different for past and future ephemerides.

Note that the NASA/JPL Navigation and Ancillary Information Facility (NAIF) provides software and files to facilitate the above transformations. This includes a Planetary Constants Kernel (PCK) made using the lunar libration information extracted from the DE/LE 403 ephemeris, and a special lunar frames kernel (FK) providing the specifications and data needed to construct the PA to ME system transformation. A new version of the PCK will also be provided when a new JPL ephemeris is released. See http://naif.jpl.nasa.gov for further information. Roncoli (2005) also provides useful information on lunar constants and coordinates, including on the differences between the ME and PA systems and on the DE403 ephemeris.

\section{Rotation elements for planets and satellites}

The rotation rate of Saturn, which is given in Table 1 is based on Voyager observations of kilometer wavelength radio signals. Recent Cassini observations (Giampieri et al. 2006) of a signal in Saturn's magnetic field gives a period of about $10 \mathrm{~h}$ and $47 \mathrm{~min}$, about $8 \mathrm{~min}$ longer than the previously determined period. At this time it is uncertain whether this is the true rotation rate or what is the physical mechanism causing the different signals (Stevenson 2006). Hence, the rotation rate of Saturn has not been changed, while more results from the Cassini mission are anticipated.

The rotation rates of Uranus and Neptune were determined from the Voyager mission in 1986 and 1989. The uncertainty of those rotation rates are such that the uncertainty of the actual rotation position is more than a complete rotation in each case.

A new model for the pole position and rotation of Mars has been proposed by Konopliv et al. (2006) based on the most recent spacecraft data. At this time, following the advice of the NASA Mars Geodesy and Cartography Working Group (Duxbury 2006), the use of this new model is not recommended for cartographic purposes. This is for a number of reasons including that for the immediate future the new model would have little if any effect on cartographic products, and also that it is expected to be significantly changed in the next few years as new data becomes available. However, users with high accuracy requirements, such as Mars gravity field determination, may wish to consider using it.

The topographic reference surface of Mars is that specified in the final MOLA Mission Experiment Gridded Data Record (MEGDR) Products (Smith et al. 2003). In particular, the 128 pixels ${ }^{\circ}$ resolution, radius and topographic surfaces are recommended, although the lower resolution versions may be used where appropriate and documented, and for the areas poleward of $\pm 88^{\circ}$ latitude.

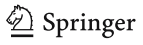


For Mercury the use of a planetocentric, east-positive (right-handed) system was adopted by the MESSENGER project more than 6 years ago to facilitate geodetic analysis, particularly topography and gravity, as well as all cartography. The Mariner 10 mission used the IAU/IAG standard system. There are standard transformations between the two coordinate sets. For the Mars Global Surveyor mission, an areocentric, east-positive system was used despite years of Mariner 4, 6, 7, and 9 and Viking data mapped with the IAU/IAG standard system.

\section{Rotational elements for minor planets and comets}

For planets and satellites, the IAU definition of north pole is the pole that lies above the invariant plane of the solar system, and the rotation can be either direct or retrograde. For minor planets and comets, given substantial indirect evidence for large precession of the rotational poles of some comets, this first definition needs to be rethought. In particular, situations exist in which the pole that is clearly "north" in the IAU sense precesses over several decades to become clearly "south" in the IAU sense. Comet 2P/Encke, which is likely to be visited by spacecraft in the foreseeable future, is a prime example of a comet for which very large precession has been inferred.

There is also clear evidence for excited state rotation at least for comet $1 \mathrm{P} / \mathrm{Halley}$ and minor planet Toutatis. In this case, the angular momentum vector moves around on the surface of the body. The rotational spin vector describes substantial excursions from the angular momentum vector during the course of the 7-day periodicity that is seen in the light curve. We can, therefore, anticipate cases in which the rotational spin pole moves back and forth between north and south on a time scale of days. Thus, there is the issue of needing to change our definition of the rotational pole.

The choice of a rotational pole for a body in simple rotation with slow precession is straightforward. One can choose the pole that follows either the right-hand rule or the left-hand rule, and the right-hand rule is chosen here. This would be the "positive" pole to avoid confusion with the north-south terminology. Ideally one would like to choose a pole for excited state rotation that reduces to this definition as the rotational energy relaxes to the ground state. For SAM (short-axis mode) rotational states, it is possible to define a body-fixed axis that circulates in a generally complex pattern about the angular momentum vector and this approaches the simple right-hand rule definition as the rotational energy relaxes to the ground state of simple rotation. Presumably the appropriate body-fixed pole is the axis of maximum moment of inertia. However, the definition for a body in a LAM (long-axis mode) rotational state is not so obvious, since there is then complete rotation about the long axis of the body as well as rotation about a short axis. In this case, the pole should be taken as the minimum moment of inertia (the long axis of an ellipsoid) according to the right-hand rule.

As specified in Sect. 6, for planets and satellites, longitude should increase monotonically for an observer fixed in inertial space. For minor planets and comets, however, with the above rule for poles, this definition corresponds always to a left-hand rule for increasing longitude, since the concept of retrograde rotation is no longer relevant. Therefore, for minor planets and comets, to be consistent with the above pole definition, increasing longitude should always follow the right-hand rule. This definition is consistent with the sense of increasing longitude used for Eros by Miller et al. (2002), 
but is inconsistent with the sense of increasing longitude used for Eros by Thomas et al. (2002).

For each minor planet and comet the positive pole of rotation is selected as the maximum or minimum moment of inertia according to whether there is short or long axis rotational state and according to the right-hand rule. So for minor planets and comets the positive pole is specified by the value of its right ascension $\alpha_{0}$ and declination $\delta_{0}$. With the pole so specified, the two intersection points of the body's equator and the ICRF equator are $\alpha_{0} \pm 90^{\circ}$. We chose one of these, $\alpha_{0}+90^{\circ}$, and define it as the node $Q$. Suppose the prime meridian has been chosen so that it crosses the body's equator at the point B. We then specify the location of the prime meridian by providing a value for W, the angle measured along the body's equator between the node $Q$ and the point B in a right-hand system with respect to the body's positive pole (see Fig. 2). The right ascension of the point $Q$ is $90^{\circ}+\alpha_{0}$ and the inclination of the body's equator to the celestial equator is $90^{\circ}-\delta_{0}$. As long as the planet, and hence its prime meridian, rotates uniformly, $W$ varies linearly with time according to the right-hand rule. In addition, $\alpha_{0}, \delta_{0}$, and $W$ may vary with time due to a precession of the axis of rotation of the body.

The angle $W$ specifies the ephemeris position of the prime meridian, and for minor planets or comets without any accurately observable fixed surface features, the adopted expression for $W$ defines the prime meridian. Where possible, however, the cartographic position of the prime meridian is defined by a suitable observable feature, and so the constants in the expression $W=W_{0}+\dot{W} d$, where $d$ is the interval in days from the standard epoch, are chosen so that the ephemeris position follows the motion of the cartographic position as closely as possible; in these cases the expression for $W$ may require emendation in the future. Table 3 gives the recommended rotation values for the direction of the positive pole of rotation and the prime meridian of selected minor planets and comets. Values are given for objects that have been imaged by spacecraft, radar, or high resolution Earth based imaging systems with sufficient resolution to establish accurate pole orientation and rotation rates. Values are not given for objects where the observations are limited to photometric light curves.

Fig. 2 Reference system used to define orientation of the minor planets and comets

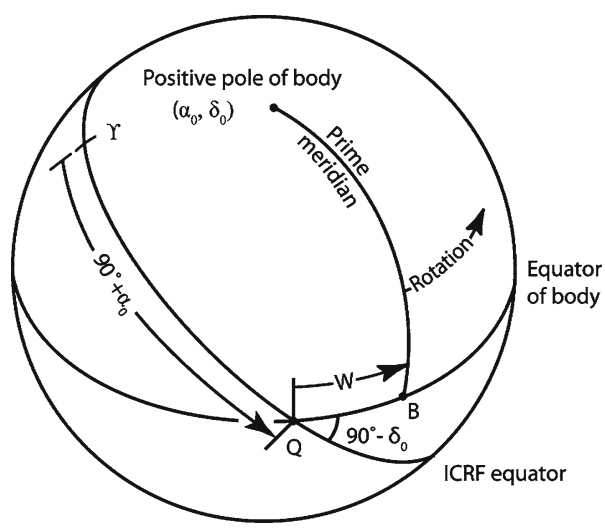


Table 3 Recommended rotation values for the direction of the positive pole of rotation and the prime meridian of selected minor planets and comets

$d$ is the interval in days from the standard epoch, i.e. J2000.0 = JD 2451545.0, i.e. 2000 January 1 12 hours TDB. $\alpha_{0}, \delta_{0}$, and $W$ are as defined in the text.

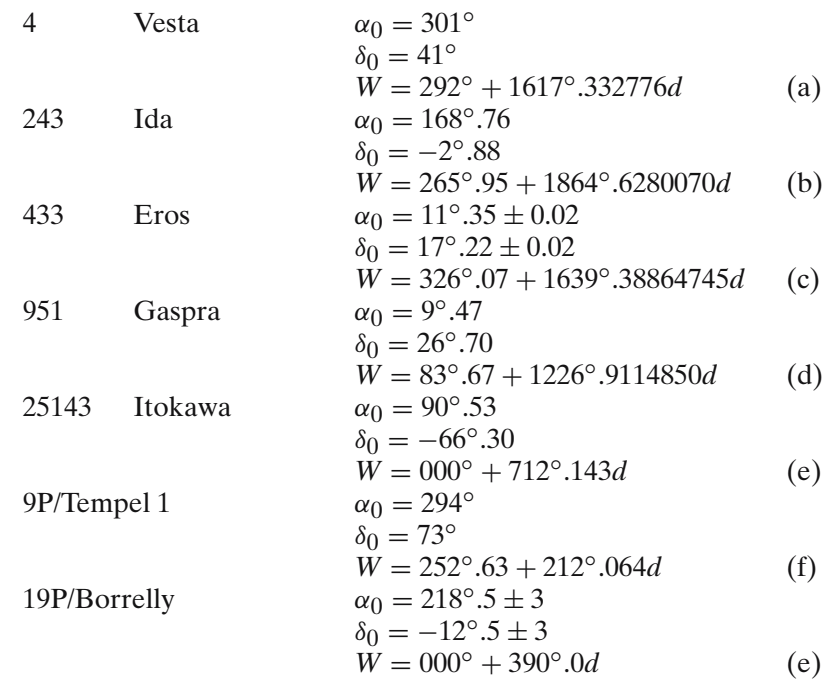

(a) The $0^{\circ}$ meridian is defined by the Olbers Regio (informal name)

(b) The $0^{\circ}$ meridian is defined by the crater Afon

(c) The $0^{\circ}$ meridian is defined by an unnamed crater

(d) The $0^{\circ}$ meridian is defined by the crater Charax

(e) Since only rotation rate information is available, the $0^{\circ}$ meridian is currently arbitrarily defined with $W_{0}=0^{\circ}$

(f) The $0^{\circ}$ meridian is defined by a $350 \mathrm{~m}$ diameter unnamed circular feature near the Deep Impactor impact site (Thomas et al. 2007a)

\section{Definition of cartographic coordinate systems for planets and satellites}

In mathematical and geodetic terminology, the terms 'latitude' and 'longitude' refer to a right-hand spherical coordinate system in which latitude is defined as the angle between a vector passing through the origin of the spherical coordinate system and the equator, and longitude is the angle between the vector and the plane of the prime meridian measured in an eastern direction. This coordinate system, together with Cartesian coordinates, is used in most planetary computations, and is sometimes called the planetocentric coordinate system. The origin is the center of mass.

Because of astronomical tradition, planetographic coordinates (those commonly used on maps) may or may not be identical with traditional spherical coordinates. Planetographic coordinates are defined by guiding principles contained in a resolution passed at the fourteenth General Assembly of the IAU in 1970. These guiding principles state that:

(1) The rotational pole of a planet or satellite which lies on the north side of the invariable plane will be called north, and northern latitudes will be designated as positive. 
(2) The planetographic longitude of the central meridian, as observed from a direction fixed with respect to an inertial system, will increase with time. The range of longitudes shall extend from $0^{\circ}$ to $360^{\circ}$.

Thus west longitudes (i.e., longitudes measured positively to the west) will be used when the rotation is direct and east longitudes (i.e., longitudes measured positively to the east) when the rotation is retrograde. The origin is the center of mass. Also because of tradition, the Earth, Sun, and Moon do not conform to this definition. Their rotations are direct and longitudes run both east and west $180^{\circ}$, or east $360^{\circ}$.

For planets and satellites, latitude is measured north and south of the equator; north latitudes are designated as positive. The planetographic latitude of a point on the reference surface is the angle between the equatorial plane and the normal to the reference surface at the point. In the planetographic system, the position of a point $(P)$ not on the reference surface is specified by the planetographic latitude of the point $\left(P^{\prime}\right)$ on the reference surface at which the normal passes through $P$ and by the height (h) of $P$ above $P^{\prime}$.

The reference surfaces for some planets (such as Earth and Mars) are ellipsoids of revolution for which the radius at the equator $(A)$ is larger than the polar semi-axis $(C)$.

Calculations of the hydrostatic shapes of some of the satellites (Io, Mimas, Enceladus, and Miranda) indicate that their reference surfaces should be triaxial ellipsoids. Triaxial ellipsoids would render many computations more complicated, especially those related to map projections. Many projections would lose their elegant and popular properties. For this reason spherical reference surfaces are frequently used in mapping programs.

Many small bodies of the solar system (satellites, minor planets, and comet nuclei) have very irregular shapes. Sometimes spherical reference surfaces are used for computational convenience, but this approach does not preserve the area or shape characteristics of common map projections. Orthographic projections often are adopted for cartographic portrayal as these preserve the irregular appearance of the body without artificial distortion. A more detailed discussion of cartographic coordinate systems for small bodies is given in Sect. 7 of this report.

Table 4 gives the size and shape parameters for the planets. In that table average (AVG), north (N), and south (S) polar radii are given for Mars. For the purpose of adopting a best-fitting ellipsoid for Mars, the average polar radius should be used - the other values are for comparison only, e.g. to illustrate the large dichotomy in shape between the northern and southern hemispheres of Mars. In applications where these differences may cause problems, the earlier recommended topographic shape model for Mars should probably be used as a reference surface.

Table 5 gives the size and shape of satellites where known. Only brightnesses are known for many of the newly discovered satellites. Poles and rotation rates are also not yet known for the new discoveries, so those satellites are not listed.

The values of the radii and axes in Tables 4 and 5 are derived by various methods and do not always refer to common definitions. Some use star or spacecraft occultation measurements, some use limb fitting, others use altimetry measurements from orbiting spacecraft, and some use control network computations. For the Earth, the spheroid refers to mean sea level, clearly a very different definition from other bodies in the Solar System.

The uncertainties in the values for the radii and axes in Tables 4 and 5 are generally those of the authors, and, as such, frequently have different meanings. Sometimes 
Table 4 Size and shape parameters of the planets

\begin{tabular}{|c|c|c|c|c|c|c|}
\hline Planet & $\begin{array}{l}\text { Mean } \\
\text { radius }(\mathrm{km})\end{array}$ & $\begin{array}{l}\text { Equatorial } \\
\text { radius }(\mathrm{km})\end{array}$ & $\begin{array}{l}\text { Polar radius } \\
(\mathrm{km})\end{array}$ & $\begin{array}{l}\text { RMS } \\
\text { deviation } \\
\text { from } \\
\text { spheroid } \\
(\mathrm{km})\end{array}$ & $\begin{array}{l}\text { Maximum } \\
\text { elevation } \\
(\mathrm{km})\end{array}$ & $\begin{array}{l}\text { Maximum } \\
\text { depression } \\
(\mathrm{km})\end{array}$ \\
\hline Mercury & $2439.7 \pm 1.0$ & Same & Same & 1 & 4.6 & 2.5 \\
\hline Venus & $6051.8 \pm 1.0$ & Same & Same & 1 & 11 & 2 \\
\hline Earth & $\begin{array}{c}6371.00 \pm \\
0.01\end{array}$ & $6378.14 \pm 0.01$ & $6356.75 \pm 0.01$ & 3.57 & 8.85 & 11.52 \\
\hline Mars & $\begin{array}{c}3389.50 \pm \\
0.2\end{array}$ & $\begin{array}{c}3396.19 \pm \\
0.1\end{array}$ & $\begin{array}{c}\text { AVG } 3376.20 \\
\pm 0.1 \\
\text { N } 3373.19 \pm \\
0.1 \\
\text { S } 3379.21 \pm \\
0.1\end{array}$ & 3.0 & $\begin{array}{c}22.64 \pm \\
0.1\end{array}$ & $\begin{array}{c}7.55 \pm \\
0.1\end{array}$ \\
\hline Jupiter* & $69911 \pm 6$ & $71492 \pm 4$ & $66854 \pm 10$ & 62.1 & 31 & 102 \\
\hline Saturn* & $58232 \pm 6$ & $60268 \pm 4$ & $54364 \pm 10$ & 102.9 & 8 & 205 \\
\hline Uranus* & $25362 \pm 7$ & $25559 \pm 4$ & $24973 \pm 20$ & 16.8 & 28 & 0 \\
\hline Neptune* & $24622 \pm 19$ & $24764 \pm 15$ & $24341 \pm 30$ & 8 & 14 & 0 \\
\hline Pluto & $1195+5$ & Same & Same & & & \\
\hline
\end{tabular}

* The radii correspond to a one-bar surface

they are standard errors of a particular data set, sometimes simply an estimate or expression of confidence. The radii and axes of the large gaseous planets, Jupiter, Saturn, Uranus, and Neptune in Table 4 refer to a one-bar-pressure surface. The radii given in the tables are not necessarily the appropriate values to be used in dynamical studies; the radius actually used to derive a value of $\mathbf{J}_{2}$ (for example) should always be used in conjunction with it.

\section{Cartographic coordinates for minor planets and comets}

For large bodies, a spherical or ellipsoidal model shape has traditionally been defined for mapping, as in our past reports. For irregularly shaped bodies the ellipsoid is obviously useless, except perhaps for dynamical studies. For very irregular bodies, the concept of a reference ellipsoid ceases to be useful for most purposes. For these bodies, topographic shapes are usually represented by a grid of radii to the surface as a function of planetocentric latitude and longitude (when possible, or also by a set of vertices and polygons).

Another problem with small bodies is that two coordinates (i.e. spherical angular measures) may not uniquely identify a point on the surface of the body. In other words it is possible to have a line from the center of the object intersect the surface more than once. This can happen on large and even mostly ellipsoidal objects such as the Earth, because of such features as overhanging cliffs and natural bridges and arches. However, on large bodies these features are relatively very small and often ignored at the scale of most topographic maps. For small bodies they may be fairly large relative to the size of the body. Example cases are on Eros (at a small patch west of Psyche), and certainly on Kleopatra (Ostro 2000), possibly on Toutatis near its 'neck', and perhaps near the south pole of Ida, some radii may intersect the surface more than once. Even on small bodies this problem is usually restricted to small areas, but it still may make a planetocentric coordinate system difficult to use. Cartographers 


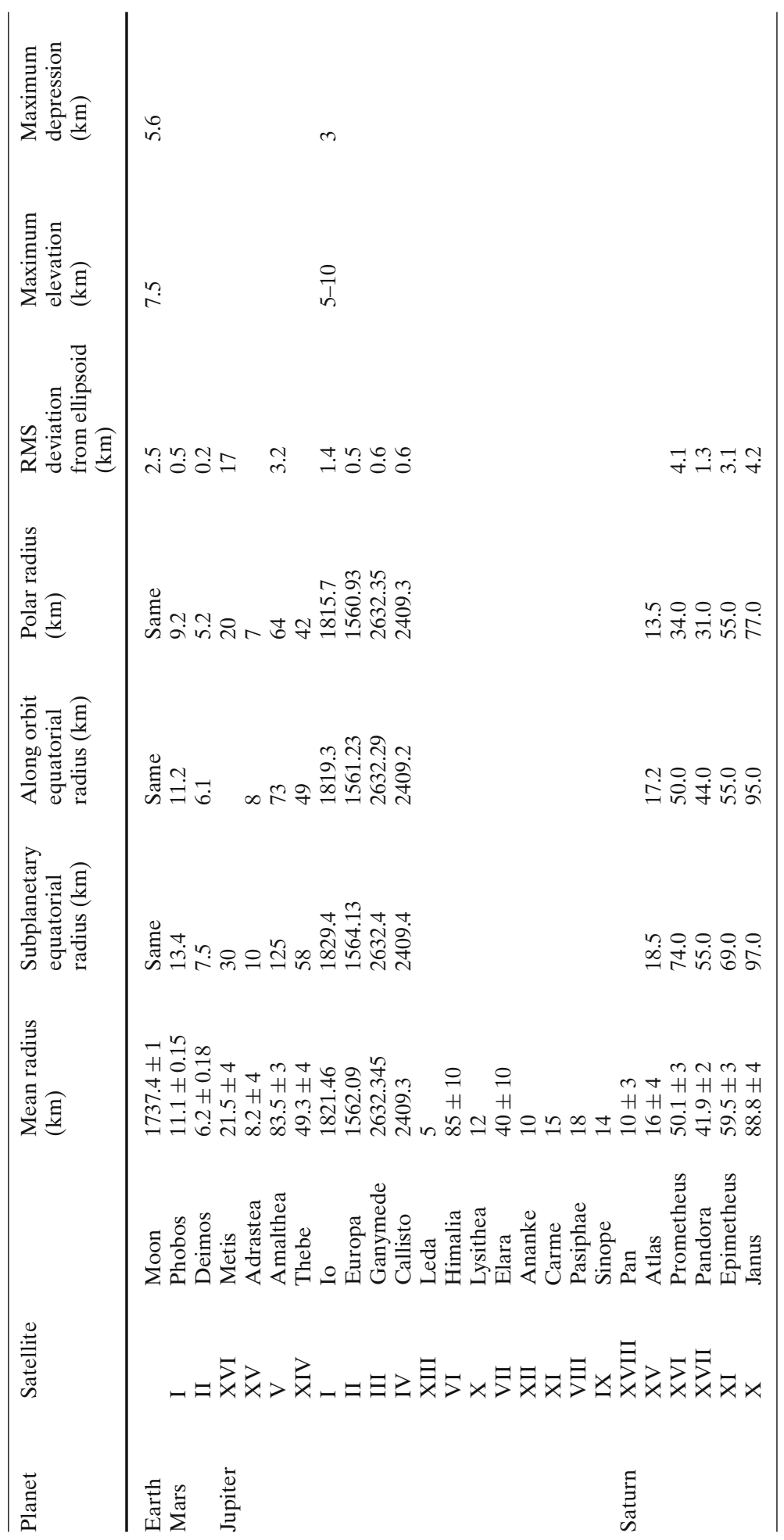

算 Springer 


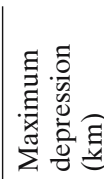

$\infty+6 \sim$

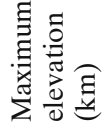

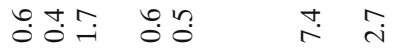

乌ి

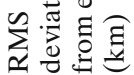

急

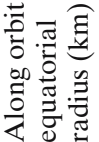

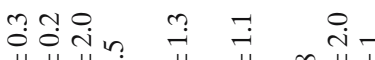

$\mathrm{H}+\mathrm{H}+\mathrm{i} \quad \mathrm{H} \infty \mathrm{H}+\mathrm{H}$

$\stackrel{-1}{-1}$

$\mathrm{H}+\mathrm{H}$

วิํํㄹ छ

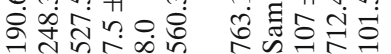

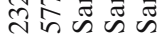

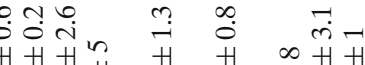

$\stackrel{0}{0}$

H H

$\infty+H$ o n

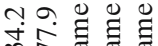

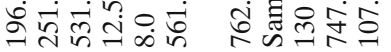

กิก

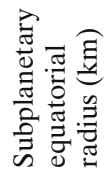

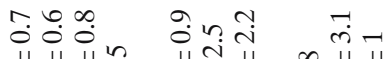

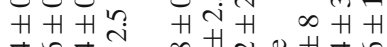

A.

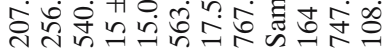

$\stackrel{0}{0} 0$

$\mathrm{H}+\mathrm{H}$

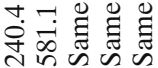

莺

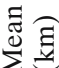

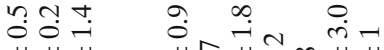

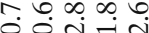

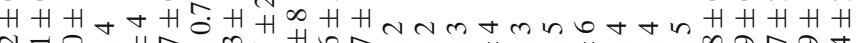

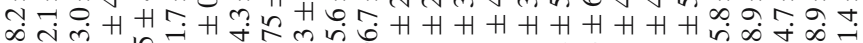

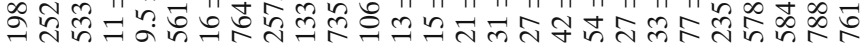

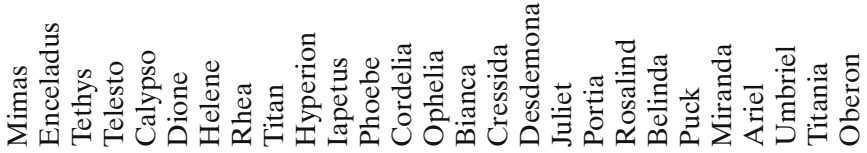

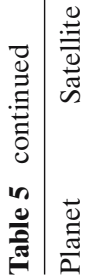

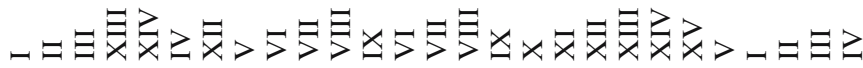




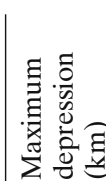

$n \stackrel{2}{2}$

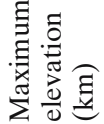

$0 \stackrel{\infty}{\sim}$

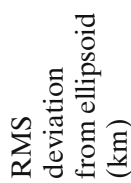

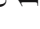

92

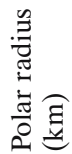

क유

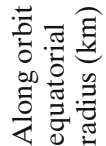

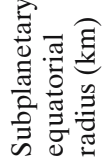

ते

$\stackrel{+\infty}{\sim}$

急

$\stackrel{4}{\mathrm{i}}$

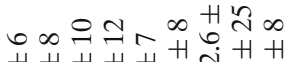

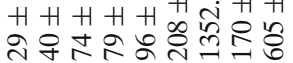

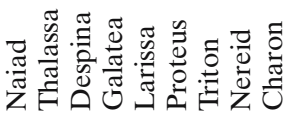

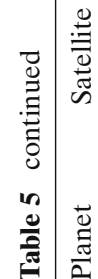

ヨコ>ラショュニー

竞

$\stackrel{\circ}{\Xi}$

型 Springer 
always have ad hoc tricks for a specific map, such as interpolating across the problem area from areas, which are uniquely defined, or by showing overlapping contours. A Cartesian or other coordinate geometry may be preferable for arbitrarily complex shapes, such as a toroidal comet nucleus, where an active region has eaten its way through the nucleus. Such coordinate geometries may also be useful for irregular bodies imaged only on one side, such as for 19P/Borrelly and 81P/Wild 2.

With the introduction of large mass storage to computer systems, digital cartography has become increasingly popular. Cartographic databases are important when considering irregularly shaped bodies and other bodies, where the surface can be described by a file containing the coordinates for each pixel. In this case the reference sphere has shrunk to a unit sphere. Other parameters such as brightness, gravity, etc., if known, can be associated with each pixel. With proper programming, pictorial and projected views of the body can then be displayed.

Taking all of this into account, our recommendation is that longitudes on minor planets and comets should be measured positively from 0 to 360 degrees using a righthand system from a designated prime meridian. The origin is the center of mass, to the extent known.

Latitude is measured positive and negative from the equator; latitudes toward the positive pole are designated as positive. For regular shaped bodies the cartographic latitude of a point on the reference surface is the angle between the equatorial plane and the normal to the reference surface at the point. In the cartographic system, the position of a point $(P)$ not on the reference surface is specified by the cartographic latitude of the point $\left(P^{\prime}\right)$ on the reference surface at which the normal passes through $P$ and by the height $(h)$ of $P$ above $P^{\prime}$.

For irregular bodies orthographic digital projections often are adopted for cartographic portrayal as these preserve the irregular appearance of the body without artificial distortion. These projections should also follow the right-hand rule.

Table 6 contains data on the size and shape of selected minor planets and comets. The first column gives the effective radius of the body and an estimate of the accuracy

Table 6 Size and shape parameters of selected minor planets and comets

\begin{tabular}{lllll}
\hline Asteroid/ comet & Effective radius $(\mathrm{km})$ & \multicolumn{4}{l}{ Radii measured along principal axes $(\mathrm{km})$} \\
\hline 1 Ceres & & $487.3 \pm 1.8$ & $454.7 \pm 1.6$ & \\
4 Vesta & & $289 \pm 5$ & $280 \pm 5$ & $229 \pm 5$ \\
243 Ida & $15.65 \pm 0.6$ & 26.8 & 12.0 & 7.6 \\
253 Mathilde & $26.5 \pm 1.3$ & 33 & 24 & 23 \\
433 Eros & $8.45 \pm 0.02$ & 17.0 & 5.5 & 5.5 \\
951 Gaspra & $6.1 \pm 0.4$ & 9.1 & 5.2 & 4.4 \\
4179 Toutatis & & 2.13 & 1.015 & 0.85 \\
25143 Itokawa & & 0.535 & 0.294 & 0.209 \\
1P/Halley & & $8.0 \pm 0.5$ & $4.0 \pm 0.25$ & $4.0 \pm 0.25$ \\
9P/Tempel 1 & $3.0 \pm 0.1$ & 3.7 & 2.5 & \\
19P/Borrelly & $4.22 \pm 0.05$ & $3.5 \pm 0.2$ & - & - \\
81P/Wild 2 & 1.975 & 2.7 & 1.9 & 1.5 \\
\hline
\end{tabular}

(a) An oblate spheroid

(b) The maximum and minimum radii are not properly the values of the principal semi-axes, they are half the maximum and minimum values of the diameter. Due to the large deviations from a simple ellipsoid, they may not correspond with measurements along the principal axes, or be orthogonal to each other 
of this measurement. This effective radius is for a sphere of equivalent volume. The next three columns give estimates of the radii measured along the three principal axes.

The uncertainties in the values for the radii in Table 6 are generally those of the authors, and, as such, frequently have different meanings. Sometimes they are standard errors of a particular data set, sometimes simply an estimate or expression of confidence.

The radii given in the tables are not necessarily the appropriate values to be used in dynamical studies; the radius actually used to derive a value for the dynamical form factor $\left(\mathrm{J}_{2}\right)$ (for example) should always be used in conjunction with it.

Acknowledgements We appreciate the efforts of Mark Rosiek, who assisted in revising the Figures. Also, Myles Standish, Jim Williams, Chuck Acton, and Ralph Roncoli have provided information on the use of the DE403 lunar ephemeris and other suggestions.

\section{Appendix: Changes since the last report}

This appendix summarizes the changes that have been made to the tables since the 2003 report (Celestial Mechanics and Dynamical Astronomy 91, 203-215, 2005).

1. The expression for the Sun's rotation has been changed to account for the light travel time and removing the aberration correction. The value in $W$ of $84.176^{\circ}$ has replaced $84.10^{\circ}$. The value $84.10^{\circ}$ is correct for the case where $\mathrm{d}$ is meant to be TT, when the light arrives at the Earth, not the moment when the light left the Sun. The $84.176^{\circ}$ is correct for the time when the light left the Sun without the aberration correction, which is consistent with the values given for the planets, whose light travel time is not as constant as for the Sun.

2. The pole and rotation rate of Pluto in Table 1 and of Charon in Table 2 have been improved based on Tholen and Buie 1997. The pole of Jupiter has been improved based on Jacobson 2005 private communication and Jacobson 2002. The Mars value of $W_{0}$ has not changed, but the correct reference is given here as Duxbury et al. 2001.

3. The expressions of the pole and rotation of the Moon are noted in Table 2 as being for low precision use. An algorithm is described in the text for using the JPL DE403 lunar ephemeris, rotated to the mean Earth/polar axis system, in order to obtain the pole and rotation with high precision.

4. The new pole and rotation models of Konopliv et al. 2006 for Mars and Giampieri et al. 2006 for Saturn are noted in the text, but not recommended for general use at this time.

5. The pole and rotation rate of Phoebe have been updated in Table 2 and the size and shape have been updated in Table 5 (P. C. Thomas private communication, also see Porco et al. 2005).

6. The pole and rotation rate of Itokawa has been added to Table 3 based on Fujiwara et al. 2006. Values for 9P/ Tempel 1 have been added to Tables 3 and 6 based on Thomas et al. 2007a.

7. In Table 5 the sizes and shapes of Saturn satellites I, II, III, IV, and V have been corrected based on Thomas et al. 2007b. The mean radius of I Mimas and the size and shape of VIII Iapeteus are from P. C. Thomas private communication. The 
radius of Charon has been updated based on the combination of Gulbis et al. 2006 and Sicardy et al. 2006.

8. In Table 6 the sizes of Ceres and Vesta have been added based on Thomas et al. 2005 and Thomas et al. 1997. The size of Itokawa is based on Fujiwara et al. 2006. Minor planets Mathilde, Eros, and Toutatis, and comets Halley, Tempel 1, and Wild 2 have been added. Minor planets Kleopatra, Golevka, Nyx, 1998JM8, and 1998ML14 have been deleted, because they were modeled from low resolution radar data, and cannot be mapped from those data.

9. The time epochs have been restated to be TDB, rather than TT, since the barycentric time is technically correct for these tables. TDB and TT are roughly equivalent in epoch and rate and to the accuracies given do not differ. Also the JD value has been given before the calendar date for clarity.

10. A sign error has been corrected in the equation for $W$ for 243 Ida in Table 3.

\section{References}

Davies, M.E., Abalakin, V.K., Cross, C.A., Duncombe, R.L., Masursky, H., Morando, B., Owen, T.C., Seidelmann, P.K., Sinclair, A.T., Wilkins, G.A., Tjuflin, Y.S.: Report of the IAU Working Group on Cartographic Coordinates and Rotational Elements of the Planets and Satellites. Celest. Mech. 22, 205-230 (1980)

Davies, M.E., Abalakin, V.K., Lieske, J.H., Seidelmann, P.K., Sinclair, A.T., Sinzi, A.M., Smith, B.A., Tjuflin, Y.S.: Report of the IAU Working Group on Cartographic Coordinates and Rotational Elements of the Planets and Satellites: 1982. Celest.Mech. 29, 309-321 (1983)

Davies, M.E., Abalakin, V.K., Bursa, M., Lederle, T., Lieske, J.H., Rapp, R.H., Seidelmann, P.K., Sinclair, A.T., Teifel, V.G., Tjuflin, Y.S.: Report of the IAU/IAG/COSPAR Working Group on Cartographic Coordinates and Rotational Elements of the Planets and Satellites: 1985. Celest. Mech. 39, 103-113 (1986)

Davies, M.E., Abalakin, V.K., Bursa, M., Hunt, G.E., Lieske, J.H., Morando, B., Rapp, R.H., Seidelmann, P.K., Sinclair, A.T., Tjuflin, Y.S.: Report of the IAU/IAG/COSPAR Working Group on Cartographic Coordinates and Rotational Elements of the Planets and Satellites: 1998. Celest. Mech. Dyn. Astron. 46, 187-204 (1989)

Davies, M.E., Abalakin, V.K., Brahic, A., Bursa, M., Chovitz, B.H., Lieske, J.H., Seidelmann, P.K., Sinclair, A.T., Tjuflin, Y.S.: Report of the IAU/IAG/COSPAR Working Group on Cartographic Coordinates and Rotational Elements of the Planets and Satellites: 1991. Celest. Mech. Dyn. Astron. 53, 377-397 (1992)

Davies, M.E., Abalakin, V.K., Bursa, M., Lieske, J.H., Morando, B., Seidelmann, P.K., Sinclair, A.T., Yallop, B., Tjuflin, Y.S.: Report of the IAU/IAG/COSPAR Working Group on Cartographic Coordinates and Rotational Elements of the Planets and Satellites: 1994. Celest. Mech. Dyn. Astron. 63, 127-148 (1996)

Davies, M.E., Colvin, T.R.: Lunar coordinates in the regions of the Apollo landers. JGR 105, (E8), 20,277-20, $280(2000)$

Duxbury, T.: Minutes of 2006 October 3 Meeting of the MGCWG, as of 2006 October 31 (2006)

Duxbury, T., et al.: Mars Geodesy/Cartography Working Group Recommendations on Mars Cartographic Constants and Coordinate Systems. In "Orientation at Epoch" page 1, column 2, http://astrogeology.usgs.gov/Projects/ISPRS/MEETINGS/Flagstaff2001/abstracts/ isprs_etm_OCT01_dux bury_A_mars_constants.pdf (2001)

Fujiwara, A., et al.: The rubble-pile asteroid Itokawa as observed by Hayabusa. Science 312, 1330-1334 (2006)

Giampieri, G., Dougherty, M.K., Smith, E.J., Russell, C.T.: A regular period for Saturn's magnetic field that may track its internal rotation. Nature 441, 62-64 (2006)

Gulbis, A.A.S., Elliot, J.L., Person, M.J., Adams, E.R., Babcock, B.A., Emilio, M., Gangestad, J.W., Kern, S.D., Kramer, E.A., Osip, D.J., Pasachoff, J.M., Souza, S.P., Tuvikene, T.: Charon's radius and atmospheric constraints from observations of a stellar occultation. Nature 439, 48-51 (2006)

Jacobson, R.A.: The orientation of the pole of Jupiter. BAAS 34. 9362002 
Konopliv, A., Yoder, C.F., Standish, E.M., Yuan, D.-N., Sjogren, W.I.: A global solution for the Mars static and seasonal gravity, Mars orientation, Phobos and Deimos masses, and Mars ephemeris. Icarus 182, 23-50 (2006)

Konopliv, A.S., Asmar, S.W., Carranza, E., Sjogren, W.L., Yuan, D.N.: Recent gravity models as a result of the Lunar Prospector mission. Icarus 150, 1-18 (2001)

Kovalevsky, J., Seidelmann, P.K.: Fundamentals of Astrometry. Cambridge University Press (2004)

Ma, C., Arias, E.F., Eubanks, T.M., Fey, A.L., Gontier, A.-M., Jacobs, C.S., Sovers, O.J., Archinal, B.A., Charlot, P.: The International Celestial Reference Frame As realized by Very Long Baseline Interferometry. Astron. J. 116, 516-546 (1998)

Miller, J.K., Konopliv, A.S., Antreasian, P.G., Bordi, J.J., Chesley, S., Helfrich, C.E., Owen, W.M., Wang, T.C., Williams, B.G., Yeomans, D.K., Scheeres, D.J.: Determination of shape, gravity, and rotational state of asteroid 433 Eros. Icarus 155, 3-17 (2002)

Ostro, S.J., Hudson, R.S., Nolan, M.C., Margot, J.-L., Scheeres, D.J., Campbell, D.B., Magri, C., Giosini, J.D., Yeomans, D.K.: Radar observations of asteroid 216 Kleopatra. Science 288, 836-839 (2000)

Peale, S.: The proximity of Mercury's spin to Cassini state 1 from adiabatic invariance. Icarus 181, 338347 (2006)

Porco, C.C., Baker, E., Barbara, J., Beurle, K., Brahic, A., Burns, J.A., Charnoz, S., Cooper, N., Dawson, D.D., Del Genio, A.D., Denk, T., Dones, L., Dyudina, U., Evans, M.W., Giese, B., Grazier, K., Helfenstein, P., Ingersoll, A.P., Jacobson, R.A., Johnson, T.V., McEwen, A., Murrray, C.D., Neukum, G., Owen, W.M., Perry, J., Roatsch, T., Spitale, J., Squyres, S., Thomas, P.C., Tiscareno, M., Turtle, E., Vasavada, A.R., Veverka, J., Wagner, R., West, R.: Cassini Imaging Science: Initial Results on Phoebe and Iapetus. Science 307, 1237-1242 (2005)

Roncoli, R.: Lunar constants and models document. JPL D-32296, available at http://ssd.jpl. nasa.gov/?lunar_doc (2005)

Seidelmann, P.K., Abalakin, V.K., Bursa, M., Davies, M.E., de Bergh, C., Lieske, J.H., Oberst, J., Simon, J.L., Standish, E.M., Stooke, P., Thomas, P.C.: Report of the IAU/IAG Working Group on Cartographic Coordinates and Rotational Elements of the Planets and Satellites: 2000. Celest. Mech. Dyn. Astron. 82, 83-110 (2002)

Seidelmann, P.K., Archinal, B.A., A’Hearn, M.F., Cruikshank, D.P., Hilton, J.L., Keller, H.U., Oberst, J., Simon, J.L., Stooke, P., Tholen, D.J., Thomas, P.C.: Report of the IAU/IAG Working Group on Cartographic Coordinates and Rotational Elements: 2003. Celest. Mech. Dyn. Astron. 91, 203215 (2005)

Sicardy, B. et al.: Charon's size and an upper limit on its atmosphere from a stellar occultation. Nature 439, 52-54 (2006)

Smith, D., Neumann, B., Arvidson, R.E., Guinness, E.A., Slavney, S.: Mars Global Surveyor Laser Altimeter Mission Experiment Gridded Data Record. NASA Planetary Data System, MGS-M-MOLA-5-MEGDR-L3-V1.0, 2003. Available on-line from http://pdsgeosciences.wustl.edu/missions/mgs/megdr.html

Stevenson, D.J.: A new spin on Saturn. Nature 441, 344-35 (2006)

Tholen, D.J., Buie, M.W.: The Orbit of Charon. Icarus 125, 245-260 (1997)

Thomas, P.C., Veverka, J., Belton, M.J.S., Hidy, A., A'Hearn, M.F., Farnham, T.L., Groussin, O., Li, J.-Y., McFadden, L.A., Sunshine, J., Wellnitz, D., Lisse, C., Schultz, P., Meech, K.J., Delamere., W.A.: The shape, topography, and geology of Tempel 1 from Deep Impact observations. Icarus 187, 4-15 (2007a)

Thomas, P.C., et al.: Shapes of the Saturnian icy satellites and their significance. Icarus, in press (2007b)

Thomas, P.C., Wm. Parker, J., McFadden, L.A., Russell, C.T., Stern, S.A., Sykes, M.V., Young, E.F.: Differentiation of the asteroid Ceres as revealed by its shape. Nature 437, 224-226 (2005)

Thomas, P.C., Joseph, J., Carcich, B., Veverka, J., Clark, B.E., Bell, J.F., Byrd, A.W., Chomko, R., Robinson, M., Murchie, S., Prockter, L., Cheng, A., Izenberg, N., Malin, M., Chapman, C., McFadden, L.A., Kirk, R., Gaffey, M., Lucey, P.G.: Eros: shape, topography, and slope processes. Icarus 155, 18-37 (2002)

Thomas, P.C., Binzel, R.P., Gaffey, M.J., Zellner, B.H., Storrs, A.D., Wells, E.N.: Vesta: spin, pole, size, and shape from HST Images. Icarus 128, 88-94 (1997) 